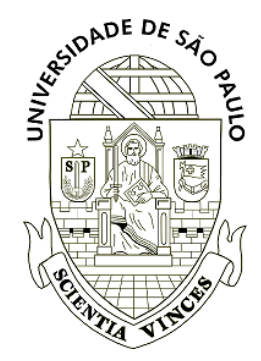

Universidade de São Paulo

Biblioteca Digital da Produção Intelectual - BDPI

Shape description based on bag of salience points

Symposium on Applied Computing, 30th, 2015, Salamanca.

http://www.producao.usp.br/handle/BDPI/49014

Downloaded from: Biblioteca Digital da Produção Intelectual - BDPI, Universidade de São Paulo 


\title{
Shape Description Based on Bag of Salience Points
}

\author{
Glauco V. Pedrosa ${ }^{1}$ Agma J. M. Traina $^{1}$ and Celia A. Z. Barcelos ${ }^{2}$ \\ ${ }^{1}$ Instituto de Ciências Matemáticas e de Computação - ICMC \\ ${ }^{1}$ University of Sao Paulo (USP), São Carlos, SP - Brazil \\ ${ }^{2}$ Federal University of Uberlandia (UFU), Uberlândia, MG - Brazil \\ \{gpedrosa, agma\}@icmc.usp.br, celiazb@ufu.br
}

\begin{abstract}
Salient points are very important for image description because they are related to the visually most important parts of the image, leading to a compact and more discriminative representation close to human perception. Based on these promising features, in this paper we propose a new shape descriptor, namely Bag-ofSalience-Points (BoSP), using the shape salience points combined with the Bag-of-Visual-Words modeling approach. Each salience point, after extracted from the shape contour, is represented by its curvature value using a multi-scale procedure proposed in this work. Taking advantage of this representation, each salience is assigned to a visual word according to a Dictionary of Curvatures. The final shape representation is given by computing a histogram of visual words detected in the shape, combined with a spatial pooling approach that encodes the distance distribution of the visual words in relation the shape centroid. This proposed new shape description allows to analyze the dissimilarity between shapes using fast distance functions, such as the City-block distance, even if two shapes have different number of salience points. This is a powerful asset to reduce the computational complexity when retrieving images. Compared to other shape descriptors, the BoSP descriptor has the advantage of proving a powerful shape description with high recognition accuracy, a compact representation invariant to geometric transformations while demanding a low computational cost to measure the dissimilarity of shapes.
\end{abstract}

\section{Categories and Subject Descriptors}

I.4 [IMAGE PROCESSING AND COMPUTER VISION]: Image Representation, Scene Analysis

\section{Keywords}

image representation; image retrieval; shape descriptor; salience point; bag-of-features; visual words

\section{INTRODUCTION}

The rapidly increasing amount of digital images have created the demand for Content-Based Image Retrieval (CBIR) systems. These

Permission to make digital or hard copies of all or part of this work for personal or classroom use is granted without fee provided that copies are not made or distributed for profit or commercial advantage and that copies bear this notice and the full citation on the first page. Copyrights for components of this work owned by others than ACM must be honored. Abstracting with credit is permitted. To copy otherwise, or republish, to post on servers or to redistribute to lists, requires prior specific permission and/or a fee. Request permissions from Permissions@acm.org.

SAC'15 April 13-17, 2015, Salamanca, Spain.

Copyright 2015 ACM 978-1-4503-3196-8/15/04\$15.00

http://dx.doi.org/10.1145/2695664.2695838 systems rely on finding similar images in the same way a search engine responds to a text query. In this context, the first and most important step to retrieve similar images is to describe the image features. Robust to image transformations, discriminative, and efficient image features can significantly improve the performance of image/video retrieval and recognition. Research in this area has advanced in order to obtain methods that capture the semantics of the image, extracting a combination of features perceptually efficient and compact [10]. However, there is still demand for faster and more invariant descriptors.

Color, shape and/or texture are the traditional information used to describe the image semantics. Shape is the visual content that represents the silhouette of objects, and it is widely exploited in CBIR systems due to its importance in semantically characterizing the content of an image close to human subjectivity. Despite the importance of shapes in the image representation, the development of a shape descriptor is a challenging task in the computer vision area. The main reason is the fact that a same object may present a rich variability of shapes and different objects may present shapes with a high visual similarity, such as the shapes illustrated in Fig. 1. A descriptor should be able to encode these subtle differences and, at the same time, being invariant to geometric variations that may occur with the shapes, such as rotation, translation and scale. Moreover, other important properties are desirable for a shape descriptor, such as: compact representation, fast computational time to extract the features and low computational cost to measure the dissimilarity.

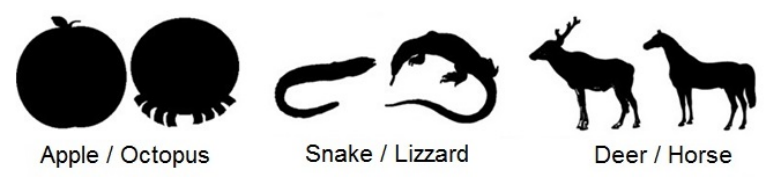

Figure 1: Shapes of different objects with high visual similarity.

Many shape descriptors have been proposed in the literature [1]. Some of these descriptors use the information extracted from the shape contour, while others use the information from the whole shape. Contour-based methods, such as Fourier Descriptors [3], is quite popular in the literature, but this approach may suffer negative effects when the contour has a lot of noise. To overcome this, region-based methods, such as Zernike Moments [4], take into account all the pixels within the shape region. A recent shape descriptor with a high neurocognitive-based inspiration, called Shape Salience Descriptor (SSD) [7, 6], takes into consideration only the salience points of the shapes. A salience point (or corner point) is a point with high curvature value in the shape contour, such as illustrated in Fig. 2, and it has a huge relevance to 
human perception. Indeed, salience points are very useful for shape characterization due to its compact representation combined with invariance to geometric transformations, such as rotation and translation.

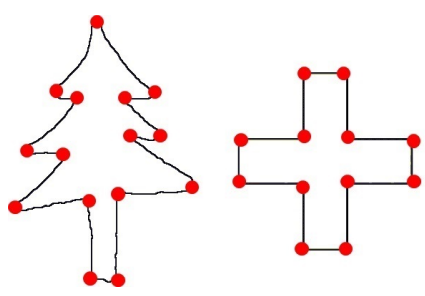

Figure 2: Salience points of some shapes.

The main drawback of using salience points as shape characterization is the computational cost for computing the dissimilarity between two shapes. This disadvantage is concerned to the fact that two shapes may have different number of salience points, thus we cannot use traditional distance functions, such as Euclidean distance, to measure the dissimilarity. The SSD, for example, uses a quadratic distance based on the minimization of a functional which considers all possible pairwise combinations between the salience points of the shapes. This fact increases the computational time to analyze the dissimilarity of shapes, affecting the effectiveness of the CBIR system.

In this paper we developed a new and effective shape descriptor called Bag-of-Salience-Points (BoSP), which is based on the use of salience points for shape representation combined with the popular Bag-of-Visual-Words (BoVW) approach [8]. In the computer vision area, the BoVW is a popular and efficient modeling approach used for image/video representation by encoding local image features into a single global representation using the idea of visual words. In the BoVW approach each local feature is assigned to a visual word according to a visual dictionary, and the final representation is based on computing a histogram of visual words detected in the image. This modeling approach gives a global representation of local image features, allowing to analyze the dissimilarity between images using simple distance functions.

In our proposed BoSP descriptor, the local features are the salience points extracted from the shape contour. To extract the salience points we use the technique proposed in [5], which is based on an evolutionary anisotropic filter robust to noise of the shape contour. Then, each salience point is represented by a multi-scale method that we will present in this paper using the curvature values computed on the shape contour. After this, each salience point is assigned to a visual word according to a Dictionary of Curvatures, previous generated. The final shape representation is the histogram of visual words, quantizing the occurrence of each visual word in the shape. However, the disadvantage of using only the histogram is the fact that it loses the spatial relationship between the visual words in the image. Thus, instead of looking only at the frequency of individual words, we also need to encode the spatial relations of visual words in the shape. In this paper we also propose an effective method to include the spatial information of the visual words into the final shape representation by encoding the distance distribution of the visual word in relation to the shape centroid.

To briefly summarize, the contributions of this paper are threefold:

- a multi-scale method to efficiently represent the salience points of a shape;

- a robust modeling approach to encode the final shape representation into a one single feature vector based on a Dictionary of Curvatures;

- a spatial pooling approach to encode the distance distribution of the visual words in the shape space.

The combination of these three proposals describes a shape in a robust and compact way, with the advantage of being able to use simple and fast distance functions to measure the dissimilarity between shapes represented by the salience points.

Experiments were made comparing the proposed shape descriptor with several other shape descriptors using public standard shapes datasets, such as the MPEG-7 CE-Shape-1 dataset [2]. The remainder of this paper is structured as follows: Section 2 explains the proposed method; Section 3 presents the experimental analysis and Section 4 gives our conclusions.

\section{PROPOSED METHOD}

In this paper, we propose the Bag-of-Salience-Points (BoSP), a new and effective shape representation using salience points as local visual attention features. Fig. 3 shows the scheme of our shape descriptor, which is summarized as follows:

1. The salience shape points are detected using an evolutionary method based on an anisotropic filter;

2. The salience points are represented in a 5-dimensional feature vector, which represent the multi-scale curvature values of the salience in the shape contour;

3. Using a Dictionary of Curvatures, each salience point is assigned to a visual word;

4. The final shape description is given by a global histogram of visual words combined with spatial-based histograms that encode the distance distribution of the visual words in the shape space.

In the next subsections we explain, in details, each step of the proposed shape descriptor.

\subsection{Saliences detection}

To extract the salience points from the shape contour, we utilize the corner detector proposed in [5]. This detector is based on a nonlinear equation diffusion to smooth the curvature value of the shape contour points. This filter is able to smooth the noisy points preserving the high curvature points that refer to a salience. In the following we will give a brief description of this corner detector.

Let the discrete shape contour $B=\left\{p_{1}, \ldots, p_{m}\right\}$ be represented by its points ordained in a clockwise direction, where $p_{i}=\left(x_{i}, y_{i}\right)$, $i=1, \ldots, m, m$ is the number of contour points, $x_{i}$ and $y_{i}$ represent the coordinates of point $p_{i}$ on the image plane. The corner detector proposed in [5] is carried out by three steps described as follows:

1. The first step consists of calculating the set $\tilde{B}=$ $\left\{\Psi_{t}\left(p_{1}\right), \ldots, \Psi_{t}\left(p_{m}\right)\right\}$, where $\Psi_{t}\left(p_{i}\right)$ represents the curvature value of point $p_{i}$. Given a contour point $p_{i}=\left(x_{i}, y_{i}\right)$ and its $t$-left and $t$-right neighbors, $p_{i+t}=$ $\left(x_{i+t}, y_{i+t}\right)$ and $p_{i-t}=\left(x_{i-t}, y_{i-t}\right)$, the curvature value can be approximated as:

$$
\Psi_{t}\left(p_{i}\right)=\frac{\alpha \varepsilon-\gamma \delta}{\left(\alpha^{2}+\gamma^{2}\right)^{3 / 2}}
$$

where, $\alpha=\left(x_{i+t}-x_{i-t}\right), \varepsilon=\left(y_{i+t}-2 y_{i}+y_{i-t}\right), \gamma=$ $\left(y_{i+t}-y_{i-t}\right)$ and $\delta=\left(x_{i+t}-2 x_{i}+x_{i-t}\right)$. 


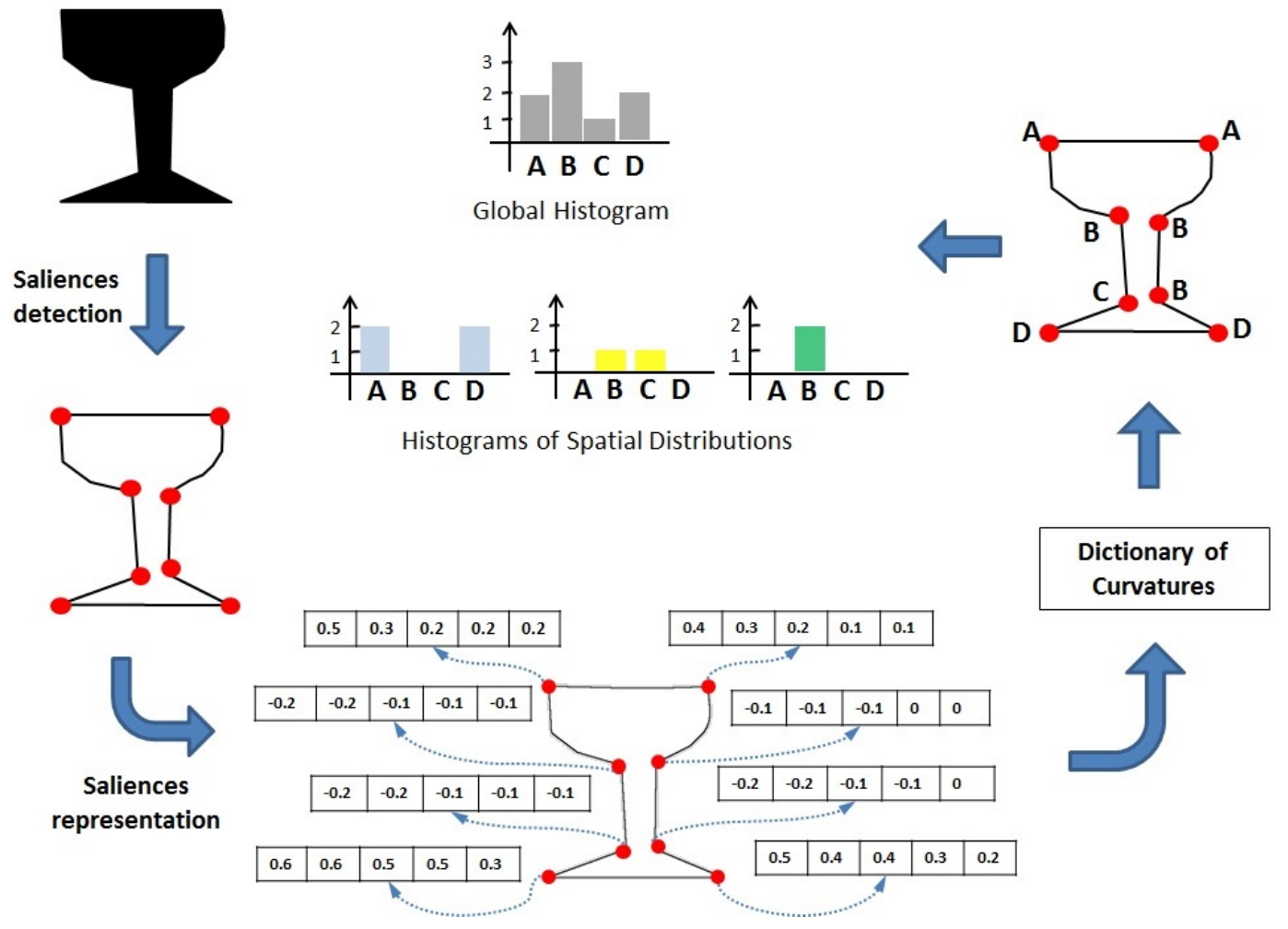

Figure 3: Scheme of the proposed method.

2. In the second step, an anisotropic filter is applied to smooth the curvature values without losing the high curvature points, which represent the salience points. Mathematically, given a function $u \subset \mathbb{R}^{d} \rightarrow \mathbb{R}, d=1,2$ or 3 , an anisotropic filter is defined as:

$$
\begin{aligned}
& u_{j}=\operatorname{div}(g(|\nabla u|) \nabla u) \\
& u_{0}=I
\end{aligned}
$$

where $g$ is a nonincreasing diffusion function, so that $g(0)$ $=1, g(\alpha) \geq 0$, and $g(\alpha) \rightarrow 0$ when $\alpha \rightarrow \infty$, and $I$ is the noisy initial signal.

3. Finally, the third step, consists of selecting the points of local maximum or local minimum which consist of the exactly localization of salience points.

After these three steps, we obtain the shape represented by its salience points: $\tilde{B}=\left\{s_{1}, \ldots, s_{n}\right\}$, where $n$ is the number of shape salience points, and $n \leq m$.

\subsection{Saliences representation}

After the detection of the salience points, we need to describe each salience with a representative value. In this paper, we propose the strategy of analyzing the contour segment where the salience is localized using a multi-scale curvature representation.

Given a point $p_{i}$ of the shape contour, to calculate the curvature of this point we can use Eq. 1. This equation is a discrete implementation of a continuous curvature equation, which needs a parameter $t$ to represent the neighborhood when computing the derivative. Varying the value of $t$ we can obtain a multi-scale representation of the shape contour. To make the representation invariant to scale, the value of $t$ should set based on the number of points in the shape contour, therefore if the size of the shape is increased or decreased, the representation is not affect by this transformation.

The multi-scale representation of each salience point is obtained using different values of $t$. In this work we propose to use 5 different values of $t=\left\{\frac{m}{\alpha}, \frac{m}{2 \alpha}, \frac{m}{3 \alpha}, \frac{m}{4 \alpha}, \frac{m}{5 \alpha}\right\}$, where $m$ is the number of points in the shape contour, $\alpha$ is a constant such that $5 \alpha<m$. Formally, let $\tilde{B}=\left\{s_{1}, \ldots, s_{n}\right\}$ be the salience points extracted from the shape using the technique described in section 2.1. Each salience point $s_{i}$ will be represented by a 5-dimensional vector that represents the curvature values computed using 5 different values of $t$, this mean, $s_{i}=\left[\Psi_{\frac{m}{\alpha}}\left(s_{i}\right), \Psi_{\frac{m}{2 \alpha}}\left(s_{i}\right), \Psi_{\frac{m}{3 \alpha}}\left(s_{i}\right), \Psi_{\frac{m}{4 \alpha}}\left(s_{i}\right), \Psi_{\frac{m}{5 \alpha}}\left(s_{i}\right)\right]$. In the experiments we $\operatorname{set} \alpha=5$.

\subsection{Obtaining the Dictionary of Curvatures}

Before assigning a visual word to each salience point, we first need to obtain a Dictionary of Curvatures. This dictionary is generated only once and it is the same for all shape representation. In the literature, clustering is a common method for learning a dictionary and the same strategy adopted in our work.

Figure 4 illustrates the process of generating the Dictionary of Curvatures. The dictionary is built by clustering the salience points extracted from a sample set of shapes in the dataset. Formally, let $P=\left\{s_{1}, s_{2}, \ldots, s_{q}\right\}$ be the salience points of this set with each salience $s_{i}$ represented by the 5 -dimensional vector presented previously. The Dictionary of Curvatures is given by a division of $P$ into $k$ distinct clusters $\pi_{k}=\left\{C_{1}, C_{2}, \ldots, C_{k}\right\}$, so that $C_{1} \cup C_{2} \cup \ldots \cup C_{k}=P, C_{i} \neq \emptyset$, and $C_{i} \cap C_{j}=\emptyset$ for $i \neq j$ and $i, j=1 \ldots k$. A visual word $w_{i}$ is the centroid of cluster $C_{i}$.

The size of the dictionary is the number $k$ of clusters provided to the clustering algorithm, where $\mathrm{k}$ is a number empirically determined. However, as will discuss in the experimental section, 

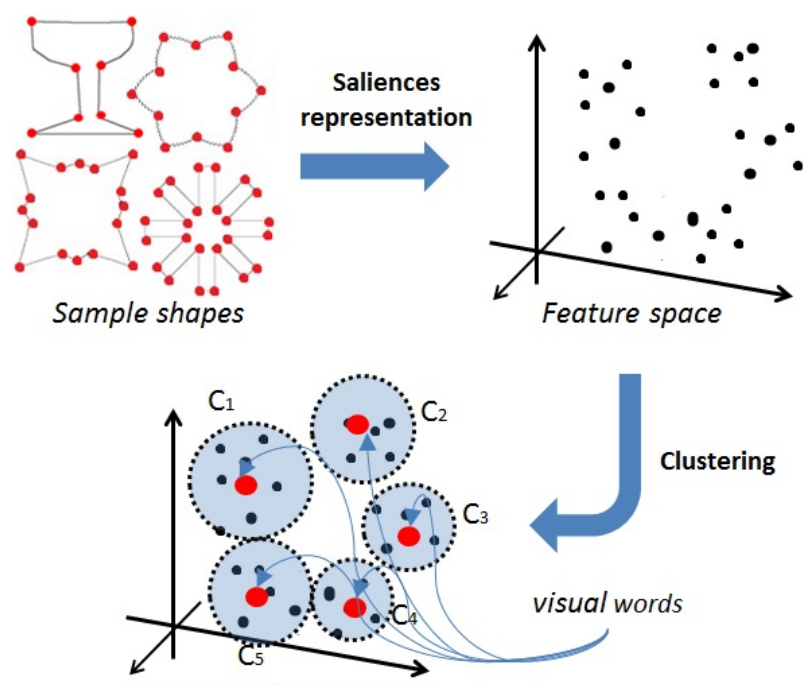

Clustered feature space

Figure 4: Constructing the Dictionary of Curvatures: the salience points of a sample of shapes are represented using the method proposed in section 2.2. The dictionary is built by clustering the feature space, and the centroid of each cluster is considered as a visual word.

the dictionary size has little effect on the quality of the shape description, but it affects the dimensionality of the final feature vector.

\subsection{Representing the salience points as visual words}

Once the Dictionary of Curvatures was generated, the next step is to assign a visual word to each salience point extracted and described using the previous steps. The visual word of a salience point $s_{i}$ is the nearest visual word $w_{j}$, for $j=1 \ldots k$, where $k$ is the number of words in the dictionary. Two different salience points of the same shape can be assigned to the same word, and a word of the dictionary may not have been assigned to any point.

The main idea of using a dictionary is to consider that the salience points are similar to textual words present in a textual document. Therefore, a shape will be composed of visual words as a textual document is composed of textual words. This analogy makes easy and simple the task of computing the dissimilarity.

\subsection{Computing the global histogram}

The final shape description is given by computing the quantity of each visual words that occurs in the shape. It means that, for each visual word $w_{j}$, for $j=1 \ldots k$, of the Dictionary of Curvatures, we count how many times this word appears in the shape, generating a histogram of visual words. If only the histogram of visual words was used to represent the shape, the dimensionality of the final feature vector would be $k$, where $k$ is the number of visual words in the dictionary.

The main limitation of using only one global histogram as the final shape description is that it captures only the first order statistics of the set of visual words, thus ignoring the fact that spatial statistics, such as distance distribution, are important for shapes recognition. For this reason, we propose an effective way to encode the spatial arrangement of the visual words and to increase final shape description.

\subsection{Encoding the spatial distribution of the visual words}

Instead of computing only one histogram, the proposed idea is to compute $Z$ histograms (for $Z>1$ ) to encode the spatial arrangement of visual words. Each histogram will represent the distance distribution of the visual words in relation to the centroid of the shape. The centroid is the geometric center point of the shape, invariant to geometric transformations, and it can be easily obtained by the mean values of the coordinates $x$ and $y$ of the shape contour points.

To compute the distance distribution, we first need to determine the distance $d$ of the farthest salience point from the centroid, to determine the border zone. Then, we define $Z$ equidistant zones of distribution, and we count the occurrences of the visual words in each zone. Figure 5 illustrates the proposed idea using $Z=3$ zones of distance distributions and considering the Euclidian distance. This strategy is very useful to distinguish shape with the same quantity of visual words, once two shapes can have the same global histogram of visual words, but not the same distance distribution of visual words.
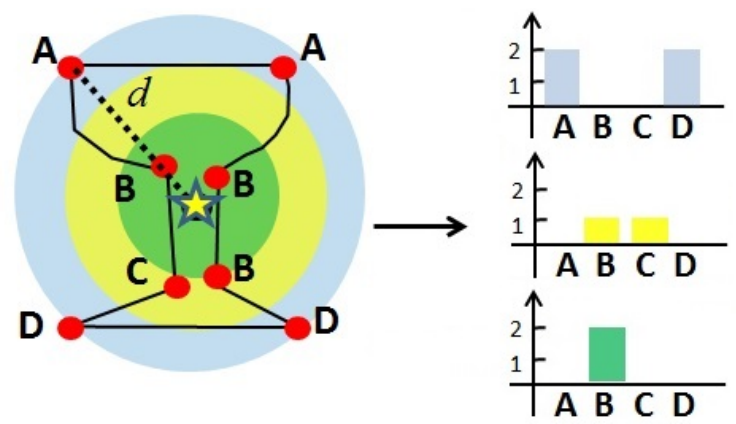

Histograms of Spatial Distributions

Figure 5: To encode the spatial arrangement of visual words, we divide the shape space in zones according to the distance from the shape centroid and we compute a histogram in each zone. In this example, we used 3 zones.

The value of $Z$ is empirically determined. However, as will show in the experiments section, for high values of $Z$ the recognition accuracy does not increase.

\subsection{Final shape representation and dissimilarity measurement}

The final feature vector that represents the final shape description is a concatenation of the global histogram and the spatial-based histograms generated in the previous step. The dimensionality of this feature vector is $k(1+Z)$, where $k$ is the quantity of words in the Dictionary of Curvatures and $Z$ the number of zones of distance distributions considered when computing the spatial-based histograms. However, the global histogram can be obtained by summing all the $Z$ spatial-based histograms. So, using this strategy the dimensionality of the final feature vector can be reduced to $k Z$.

As all the feature vectors have the same dimensionality, we can now use traditional distance functions to measure the dissimilarity. In the experiments we use the City-block distance, because it is computationally faster than the Euclidian distance.

\section{EXPERIMENTAL EVALUATION}

The proposed BoSP descriptor were compared with four other 
representative shape descriptors from the literature: MI (Moment Invariants), Fourier (Fourier Descriptor), MS Fractal (Multi-scale Fractal Dimension) [9] and the SSD (Shape Salience Descriptor) [7]. The performance of these descriptors were evaluated for shape retrieval using two standard datasets of shapes:

- MPEG-7 CE-Shape-1 dataset [2]: this is the most used and evaluated dataset of shapes. It is composed of 1400 shapes of different types, composed of 70 classes of shapes with 20 examples in each class. The shape recognition on this database is not a simple task, once there are samples that are visually dissimilar from other members of their own class. Furthermore, there are shapes that are highly similar to examples of other classes;

- Kimia-216: is composed of 18 classes each of which encompassing 12 shapes, where some of them are partially occluded. It contains silhouettes of birds, bones, brick, camels, car, children, classic cards, elephant, among others.

Our proposed descriptor has two parameters to be set: the dictionary size $k$ and the number $Z$ of zones of distance distributions. To investigate the best values of these two parameters, we exhaustively exploited different values, and Figures 6-a and 6-b show the mAP (mean Average Precision) achieved in this experiment. Analyzing the results, a dictionary size higher than 20 did not achieve an overall improvement in the accuracy. We can also observe that the quantity of zones interferes in the accuracy, however a quantify of zones higher than 4 did not improve the results. So, based on these experiments, the appropriate values of $k$ and $Z$ were set as 15 and 4, respectively.

Table 1 compares the feature vector dimensionality used by the evaluated shape descriptors. The MI descriptor has the smallest vector, but in some cases, the SSD may have the smallest one, because it depends on the number of salience points in the shape. Thus, if a shape has a number of salience points less than 7, the SSD will have the smallest vector. However, once the feature vector of SSD varies on size, it utilizes a specific distance function to compute the dissimilarity, which is not computationally efficient. In fact, this is the main drawback of the SSD descriptor, it is the slowest among all the evaluated descriptors to compute the dissimilarity of shapes, such as shown in Fig. 7. The Cityblock distance was used in all evaluated descriptors to compute the dissimilarity, except in the SSD. The proposed BoSP descriptor is the second faster descriptor to compute the dissimilarity of shapes among the evaluated descriptors.

Table 1: Feature vector dimensionality comparison, where $n$ represents the number of salience points of the shape.

\begin{tabular}{|c|c|}
\hline Descriptor & Dimensionality \\
\hline MI & 14 \\
\hline Fourier & 126 \\
\hline MS-Fractal & 100 \\
\hline SSD & $2 n$ \\
\hline BoSP & 60 \\
\hline
\end{tabular}

The comparative retrieval performance was evaluated using the Precision vs. Recall (PR). Precision is the ratio between the number of relevant retrieved images and the total number of retrieved images; and Recall is the ratio between the number of relevant retrieved images and the number of relevant images in the database. The PR curve, indicates the commitment between the two measures and, the higher the curve in the graph the better effectiveness.

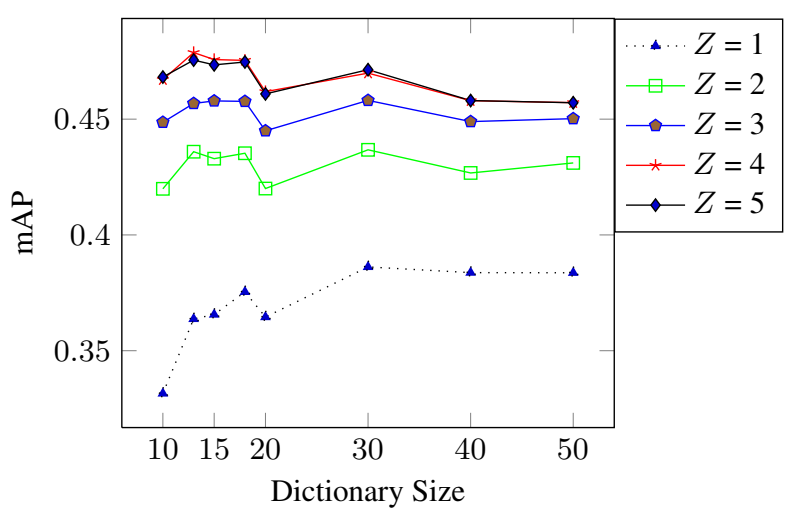

(a)

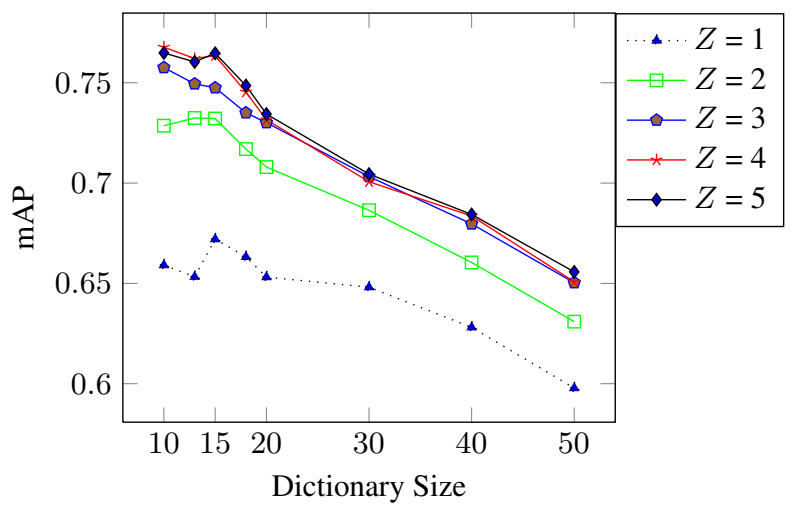

(b)

Figure 6: The mAP (mean Average Precision) values achieved using different dictionary sizes and quantity of zones in: (a) MPEG-7 dataset (b) Kimia-216 dataset. For $Z=1$ we consider only the global histogram.

Regarding the precision accuracy for retrieving shapes, Fig. 8$\mathrm{a}$ and 8-b show the PR curves of the evaluated descriptors. The proposed BoSP descriptor achieved similar performance to the SSD descriptor, but being $53 \%$ faster when computing the dissimilarity of shapes. The fastest descriptor was the Moment Invariant (MI), however it did not achieve good precision in recognizing similar shapes, negatively affecting the PR curve.

\section{CONCLUSION}

This paper proposed a new descriptor, called Bag-of-SaliencePoints (BoSP), for retrieving shape-based images using salience points as feature description. Salience points are very useful in image description because they encode the most important parts of the image in a compact way and invariant to geometric transformations.

Once two different shapes may have different number of salience points, the proposed approach uses the idea of Bag-of-VisualWords to represent the salience points of a shape into a one single feature vector. This strategy allows to compute the dissimilarity of shapes using traditional distance functions, such as Euclidian distance or City-block distance.

The strategy employed by the BoSP descriptor is to consider each type of salience point as a visual word according to a multiscale salience representation and a Dictionary of Curvature, both approaches were proposed in this work. Then, we count the 


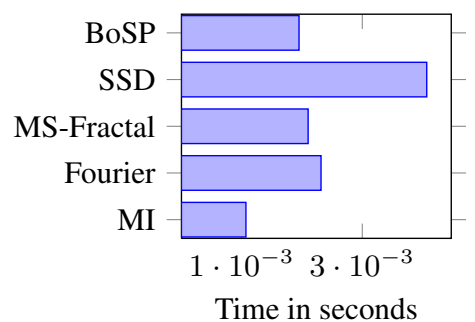

Figure 7: Average computational time to compute the dissimilarity of 2 shapes.

number of visual words that occur in the shape as the final shape description. To increase the final representation, we also proposed to use spatial-histograms that encode the distance distribution of visual words in relation to the shape centroid. These spatialhistograms yield a more robust description, once two shapes can have the same global histogram, but not the same distribution of visual words.

The proposed shape descriptor was evaluated using two representative datasets and compared to several other shape descriptors from the literature. Experimental results show that the proposed descriptor achieved the best performance while requiring a low computational cost to measure the dissimilarity. The experimental results indicate that the BoSP is a powerful and fast shape descriptor which can contribute to the content-based image retrieval field.

\section{Acknowledgements}

This work is supported, in part, by FAPESP, CAPES, SticAMSUD, the RESCUER project, funded by the European Commission (Grant 614154) and by the Brazilian National Council for Scientific and Technological Development CNPq/MCTI.

\section{REFERENCES}

[1] P. Arulmozhi and S. Abirami. Shape based image retrieval: A review. Int. Journal on Comp. Science and Engineering, 6:14-153, 2014.

[2] M. Bober. Mpeg-7 visual shape descriptors. IEEE Transactions on Circuits and Systems for Video Technology, 11(6):716-719, 2001.

[3] C. Dalitz, C. Brandt, S. Goebbels, and D. Kolanus. Fourier descriptors for broken shapes. EURASIP Journal on Advances in Signal Processing, pages 161-161, 2013.

[4] Y. Mei and D. Androutsos. Robust affine invariant region-based shape descriptors: The ica zernike moment shape descriptor and the whitening zernike moment shape descriptor. IEEE Signal Processing Letters, 16(10):877-880, Oct 2009.

[5] G. V. Pedrosa and C. A. Z. Barcelos. Anisotropic diffusion for effective shape corner point detection. Pattern Recognition Letters, 31(12):1658-1664, 2010.

[6] G. V. Pedrosa, C. A. Z. Barcelos, and M. A. Batista. A shape contour descriptor based on salience points. In IEEE International Conference on Image Processing (ICIP), pages 1049-1052, Sept 2011.

[7] G. V. Pedrosa, M. A. Batista, and C. A. Z. Barcelos. Image feature descriptor based on shape salience points. Neurocomputing, 120:156-163, 2013.

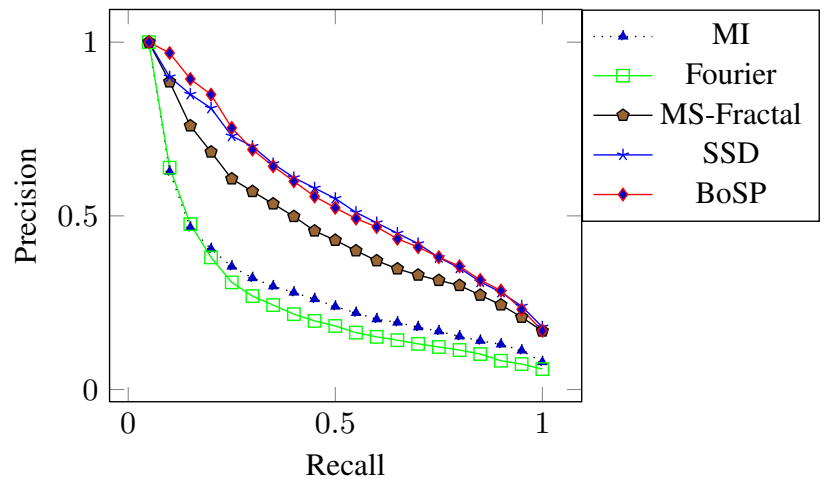

(a)

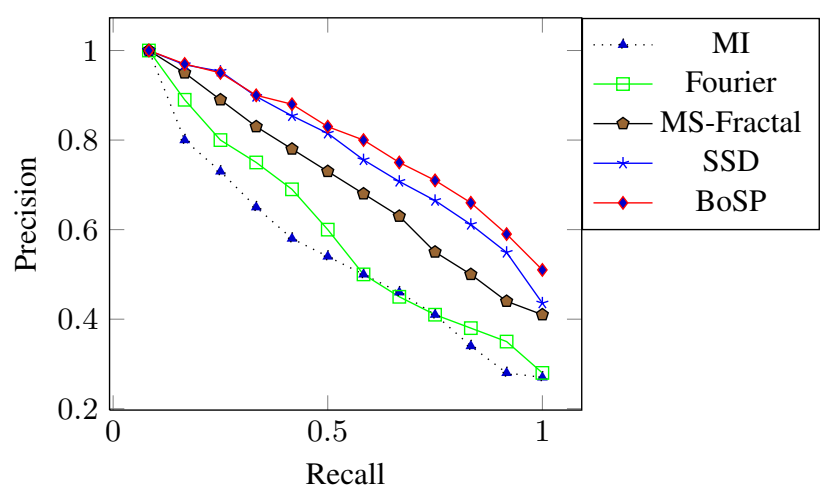

(b)

Figure 8: The PR curve for: (a) MPEG-7 dataset (b) Kimia-216 dataset

[8] J. Sivic and A. Zisserman. Video google: A text retrieval approach to object matching in videos. In IEEE International Conference on Computer Vision, pages 1470-1477, 2003.

[9] R. Torres, A. Falcão, and L. da F. Costa. A graph-based approach for multiscale shape analysis. Pattern Recognition, 37(6):1163-1174, 2003.

[10] H.-Y. Yang, Y.-W. Li, W.-Y. Li, X.-Y. Wang, and F.-Y. Yang. Content-based image retrieval using local visual attention feature. Journal of Visual Comm. and Image Representation, 25(6):1308 - 1323, 2014. 